\title{
Prognostic biomarkers in acute coronary syndrome
}

\author{
Gian Luca Salvagno', Chiara Pavan ${ }^{2}$ \\ ${ }^{1}$ Section of Clinical Biochemistry, University of Verona, Verona, Italy; ${ }^{2}$ Geriatric Medicine Division, Ospedale Mater Salutis, Legnago, Verona, Italy \\ Contributions: (I) Conception and design: GL Salvagno; (II) Administrative support: None; (III) Provision of study materials or patients: None; (IV) \\ Collection and assembly of data: GL Salvagno; (V) Data analysis and interpretation: All authors; (VI) Manuscript writing: All Authors; (VII) Final \\ approval of manuscript: All authors. \\ Correspondence to: Prof. Gian Luca Salvagno, MD, PhD. Sezione di Biochimica Clinica, Dipartimento di Neuroscienze, Biomedicina e Movimento, \\ Università degli Studi di Verona, Ospedale Policlinico G.B. Rossi, Piazzale Scuro, 10, 37134, Verona, Italy. Email: gianluca.salvagno@univr.it.
}

\begin{abstract}
The acute coronary syndrome (ACS) is a leading cause of death around the globe. Beside a still high mortality rate, additional complications of ACS include arrhythmias, left ventricular mural thrombus, cardiac fibrosis, heart failure (HF), cardiogenic shock, mitral valve dysfunction, aneurysms, up to cardiac rupture. Despite many prognostic tools have been developed over the past decades, efforts are still ongoing to identify reliable and predictive biomarkers, which may help predict the prognosis of these patients and especially the risk of HF. Recent evidence suggests that the value of a discrete number of biomarkers of myocardial fibrosis, namely the soluble form of suppression of tumorigenicity 2 (sST2) and galectin-3 (GAL-3), may be predictive of HF and death in patients with ACS. Interestingly, the already promising predictive value of these biomarkers when measured alone was shown to be consistently magnified when combined with other and well-established cardiac biomarkers such natriuretic peptides and cardiac troponins. This article is hence aimed to review the current knowledge about cardiac biomarkers of fibrosis and adverse remodeling.
\end{abstract}

Keywords: Acute coronary syndrome (ACS); prognostic biomarkers; galectin-3 (GAL-3); suppression of tumorigenicity 2 (ST-2); growth differentiation factor-15 (GDF-15)

Submitted Jun 20, 2016. Accepted for publication Jun 27, 2016.

doi: $10.21037 / \mathrm{atm} .2016 .06 .36$

View this article at: http://dx.doi.org/10.21037/atm.2016.06.36

\section{Introduction}

The acute coronary syndrome (ACS) encompasses a spectrum of unstable coronary artery disease, from unstable angina to transmural myocardial infarction. Despite remarkable progress in diagnosing patients with ACS, the identification of myocardial ischemia is still challenging, and physicians continue to admit the overwhelming majority of patients, often overestimating the likelihood of myocardial ischemia in low-risk patients (1). The American College of Cardiology and the American Heart Association (ACC/AHA) guidelines recommend a model based on the Framingham Risk Score using generally similar parameters (2). However, these current risk prediction models only provide a rough estimate of individual risk. Therefore, great value is posited in the identification and development of new biomarkers for cardiovascular (CV) risk prediction (3).
Considerable advances in the clinical laboratory testing (i.e., cardiac-specific troponin) combined with improvement in analytical techniques over the past decade have greatly improve the role of clinical biochemistry in the management of ACS (4). The use of biomarkers for disease prediction and prognosis has demonstrated potential importance for identifying groups at higher risk who may benefit from more intensive prevention and treatment $(5,6)$. Biomarkers might help in risk stratification and may guide the appropriate use of resources and therapy. The CV scientists showed that improvement of risk prediction requires a thoughtful understanding of disease mechanisms. The considerable progress achieved in the 'omics' field has successfully improved the understanding of CVD pathophysiology by comprehensively investigating disease states at the molecular level (3). As in other human disorders, the identification of biomarkers that may help predict the risk of acute 
Table 1 Biochemical prognostic markers for the evaluation and management of patients with suspected acute coronary syndrome (ACS) (7)

Biomarkers of cardiac fibrosis and adverse remodeling
Galectin-3
Suppression of tumorigenicity 2 (ST2)
Cytokines and growth factors
Growth differentiation factor-15 (GDF-15)
Osteoprotegerin
Osteopontin
Adiponectin
Matrix metalloproteinase-2 (MMP-2)
Matrix metalloproteinase-8 (MMP-8)
Matrix metalloproteinase-9 (MMP-9)
Interleukin-6
Cardiotrophin-1
Glycoprotein 130 (gp130)
C-reactive protein (CRP)
Biomarkers of neurohormonal activation
Copeptin
Extra-cardiac biomarkers
Cystatin C
Red blood cell distribution width (RDW)

coronary disease and related complications is an appealing perspective and it may also provide further insights into the pathophysiology of this disease for identifying novel targets for therapy. The prognostic significance of several clinical biomarkers in patients with ACS has been well established, and their measurement is now endorsed by the most important guidelines and recommendations for diagnosis and management of this condition (7).

Among the various biomarkers, cardiospecific troponin(s) and natriuretic peptides provide the most useful predictive information, meaning that their measurement in risk stratification and diagnosis, so that their role in the management of ACS is now endorsed by the most influential recent guidelines (8). Several biomarkers (see Table 1) have been studied in the recent year such as galectin-3 (GAL-3), suppression of tumorigenicity 2 (ST-2), growth differentiation factor-15 (GDF-15), osteoprotegerin, osteopontin, neopterin, adiponectin, matrix metalloproteinase-2 (MMP-2), matrix metalloproteinase-8 (MMP-8), matrix metalloproteinase-9 (MMP-9), interleukin-6 (IL-6), cardiotrophin-1, glycoprotein 130 (gp130), C-reactive protein (CRP), copeptin, cystatin
C as well as red blood cell distribution width (RDW) (7). However growing research findings outlined the importance of the new biomarkers of cardiac fibrosis and adverse remodeling such as GAL-3 and ST-2 $(5,9)$.

This article will review current knowledge about new clinical laboratory findings in the $\mathrm{CV}$ field, especially focused on cardiac biomarkers of fibrosis and adverse remodeling such as GAL-3, ST-2 and GDF-15.

\section{GAL-3}

GAL-3 is an emerging biomarker which has been linked to tissue fibrosis, a hallmark in cardiac remodeling and heart failure (HF). This biomarker can reliably be measured in the circulation, and several recent studies have shown its prognostic value in acute and chronic HF, and its potential utility in the general population (10). GAL-3 [also occasionally referred as Mac-2 or carbohydrate-binding protein (CBP)-35] is a $31-\mathrm{kDa}$ member of a growing family of lectins that bind $\beta$-galactosides by either $\mathrm{N}$-linked or O-linked glycosylation through their carbohydrate recognition domain (CRD) (11). The CRD sequence elements consist typically of about 130 amino acids arranged in a tightly folded conserved $\beta$-sandwich structure formed by a six strand sheet (S1-S6) and a five strand sheet (F1-F5), with the conserved carbohydrate-binding amino acids in strands S4-S6 (12). Some galectins contain just one CRD (galectins 1, 2, 5, 7, 10, 11, 13, 14, 15), others contain two homologous CRDs in a single polypeptide chain, separated by a linker of up to 70 amino acids, whereas GAL-3 contains a non-lectin N-terminal region (about 120 amino acids) connected to a CRD, often referred to as a chimera-like galectin (13). Firstly discovered in 1976, 15 galectin members have been identified and they have been classified into three subgroups depending on their structural differences and on the number of CRD within their polypeptide chains (14). All the members of this family have been numbered according to their order of discovery (i.e., from GAL-1 to GAL-15). Galectins form a family of structurally related carbohydrate binding proteins (lectins) that have been identified in a large variety of metazoan phyla. Lectins have been shown to play roles in many biological events, such as embryogenesis, adhesion and proliferation of cells, apoptosis, mRNA splicing, bacterial colonization and modulation of the immune response (15). Interestingly, galectins are expressed in vertebrates (fishes, amphibians, birds, reptiles and mammals), but they were also been found in invertebrates (worms and insects). Galectins 
originated more than 800 million years ago and are now widely distributed in the animal kingdom, from nematodes to vertebrates, conserving their basic molecular properties such as specificity and protein architecture (16). Galectins are considered to decipher glycocodes. They recognize certain sugar structures and have specific activities in given circumstances. Glycocodes are extremely different from the codes written in either nucleotides or amino acids and have hence been poorly understood (17). In 1994 galectins were defined as a family of proteins sharing conserved motifs for such core sequences, as well as the ability to specifically bind galactoside sugars (18). In vertebrates galectins were found in a variety of tissues and cells such as skin, muscle, brain, intestine, liver, kidney, placenta, cultured fibroblast and many tumor cells. A number of observations indicated their possible involvement in a variety of important phenomena occurring in multicellular animals (i.e., development, differentiation, morphogenesis, immunity, apoptosis, etc.) (16).

GAL-3 is the unique chimera-like galectin in the family having an extra long and flexible $\mathrm{N}$-terminal domain consisting of 100-150 amino acid residues, according to species of origin, made up of repetitive sequence of nine amino (11). This means that GAL-3 consists of carbohydrate recognition and collagen-like domains, which makes it able to interact with a wide array of extracellular matrix proteins, carbohydrates (e.g., N-acetyllactosamine), and unglycosylated molecules, such as cell surface receptors, extracellular receptor and glycosylated proteins of the matrix, including laminin, fibronectin, and tenascin $(11,19)$. GAL-3 has been detected in the cytoplasm, nucleus as well as in the extracellular space. There is abundant evidence for its secretion from the cytosol via non classical pathways, translocation to the nucleus or to other cellular compartments. Cytoplasmic GAL-3 has an anti-apoptotic activity, since it regulates several signal transduction pathways. Nuclear GAL-3 has been associated with pre-mRNA splicing and gene expression. It is also able to induce like cell growth, adhesion, migration, invasion, angiogenesis, immune function, apoptosis and endocytosis (11). GAL-3 has been detected in activated macrophages, eosinophils, neutrophils, mast cells, the epithelium of the gastrointestinal and respiratory tracts, the kidneys and some sensory neurons (16). The molecule is involved in various biological phenomena including cell growth, adhesion, differentiation, angiogenesis and apoptosis (20,21). More recently, Salvagno et al. provided the first evidence that the serum value of GAL-3 was remarkably increased after a $60-\mathrm{km}$ ultramarathon run, and it is hence plausible that such a remarkable increase of expression and release into circulation may promote a deleterious mechanism of fibrosis that may involve both skeletal and cardiac muscles (22).

GAL-3 is also involved in many pathological processes, including inflammation, tumor growth, and fibrosis (23). Recent research revealed that this protein is associated with several steps of invasion and metastasis, like angiogenesis, cell-matrix interaction, dissemination through blood flow and extravasation (24). GAL-3 is expressed widely in epithelial and immune cells and its expression is correlated with cancer aggressiveness and metastasis. Moreover, enhanced production and release of GAL-3 has been demonstrated in a number of inflammatory and fibrotic conditions including chronic pancreatitis, cirrhosis and lung fibrosis, as well as in patients with HF (25). Convincing evidence also suggests that GAL-3 may not be a simple bystander, but also a key player in the pathogenesis of fibrosis (26). More recently, several authors investigated the clinical utility of GAL-3 in CVD.

Sharma et al. first showed that GAL-3 is overexpressed by macrophages at an early stage of myocardium dysfunction, even before the onset of $\mathrm{HF}$, and continuous infusion of recombinant GAL-3 in mice triggers cardiac fibroblast proliferation, collagen deposition, thus ultimately causing ventricular dysfunction (27).

Expression of GAL-3 has been detected in several tissues, albeit its synthesis is substantially amplified by a number of conditions, which also include HF, lung fibrosis, kidney disease (22). Interestingly, GAL-3 is not only being used as a reliable biomarker of cardiac dysfunction and adverse outcomes, but it is also directly implicated in a kaleidoscope of biological pathways that contribute to development and worsening of $\mathrm{HF}$, thus including myofibroblast proliferation, collagen deposition and adverse cardiac remodeling (22).

Several lines of evidence convincingly suggest that the measurement of GAL-3 may be helpful in risk assessment of cardiac fibrosis and HF, both in the general population (28), and in patients with acute myocardial infarction (29).

GAL-3 can be reliably measured in plasma and several studies on plasma GAL-3 as a biomarker in HF have hence been published (30). Most of these articles focused on the role of the protein in HF, revealing that GAL-3 can be convincingly associated with mortality in patients with acute and chronic HF (30), as well as in the general population (31).

Plaque rupture is thought to develop from intimal inflammation leading to weakening of the protective fibrous cap. It has been suggested that excessive inflammatory responses are pivotal in the initiation and development 
of premature atherosclerotic lesions. Therefore, systemic markers of inflammation might identify individuals at high risk for acute coronary events in this patient population (32). Accordingly, plasma GAL-3 level has been shown to be the best short-term predictor of events in patients with HF, which led to incorporation of this measurement in the current AHA HF guidelines for risk stratification purposes of such patient $(33,34)$. Maiolino et al. also reported that GAL-3 may contribute more substantively to plaque destabilization than to plaque growth (35). Lippi et al. showed that the serum concentration of GAL-3 is virtually independent from gender, renal function, serum bilirubin and glucose in patients admitted to emergency department without evidence of ACS, and seems thereby scarcely influenced by many metabolic abnormalities that would influence additional biomarker of $\mathrm{HF}$ and cardiac fibrosis. They could also conclude that a definitive link may exist between RDW and GAL-3, so that these biomarkers may provide a synergistic information for risk assessment of patients with cardiac dysfunction in the emergency room (36).

Despite a large number of studies designed to assess the prognostic role of GAL-3 in patients with established $\mathrm{HF}$, there is limited information on its role as a prognostic biomarker in acute coronary disease. Winter et al. measured GAL-3 in 144 patients, demonstrating that patients with myocardial infarction at a very young age ( $\leq 40$ years) display increased circulating levels of this biomarker during the acute phase of myocardial infarction (32).

Falcone et al. measured GAL-3 in 125 patients with coronary artery disease. They stated that GAL-3 could be a useful biomarker of atherosclerotic plaque and in particular of its destabilization (37). Gucuk Ipek et al. measured GAL-3 in 19 patients with ACS describing that GAL-3 levels were significantly higher in ACS group than those of control group (38). Finally, Singsaas, measuring GAL-3 in 90 patients, did not find any relationship between GAL-3 levels and acute ischemic myocardial injury (39).

\section{ST2}

ST2 is an IL-1 receptor family member with transmembrane (ST2L) and soluble isoforms (sST2) (40). ST-2 is a novel and promising biomarker in the evaluation of prognosis for ACS and CV disease (41). Originally identified in 1989 as IL-1 receptor family member by two independent research group working on stimulated fibroblasts, its downstream effects include activation of T-helper type 2 (Th2) cells and production of Th2-associated cytokines. Located on chromosome 2, the human $S T 2$ gene encodes at least three isoforms by alternative promotor splicing, i.e., a membrane bound receptor ST2L, a secreted soluble form SST2 and a variant form (40).

The blood concentration of sST2 is increased in inflammatory and heart diseases, and has emerged as a clinically useful prognostic biomarker in patients with CVD, and acute dyspnea $(7,42)$. ST2 expression is upregulated after myocardial ischemia or mechanical stress, and plays a role in cardiac remodeling after ischemic injury, which make it a promising prognostic biomarker to predict future clinical HF in patients with myocardial ischemia (43). In contrast to its limited value as a diagnostic marker in ACS, sST2 seems to be an excellent prognostic marker in various cardiac diseases such as ST-elevation myocardial infarction (STEMI), nonST-elevation myocardial infarction (NSTEMI), coronary bypass and heart surgery, stable coronary artery disease, valvular disease and cardiomyopathy, acute $\mathrm{HF}$, chronic $\mathrm{HF}$, acute cardiac allograft rejection, acute Kawasaki disease (40). A role for ST2 in CVD, including ventricular remodeling and $\mathrm{HF}$ progression, has been suggested by both experimental and clinical studies.

Weinberg et al. were the first who showed increased sST2 levels in patients after myocardial infarction, with values of this biomarker positively correlating with peak creatine kinase (CK) and negatively with left ventricle ejection fraction (43).

Demyanets et al. studied 373 patients, 178 of whom with stable angina, 97 with NSTEMI and 98 with STEMI. They found that sST2 levels were significantly increased in patients with ACS as compared to patients with stable coronary artery disease and control individuals without significant stenosis on coronary angiography (44). The same research group showed that the level of circulating SST2 is associated with the stage of coronary artery disease and is increased continuously from stable coronary artery disease to NSTEMI and STEMI.

The LURIC study, a prospective investigation designed to assess biochemical and genetic risk factors for coronary artery disease in a hospital-based cohort of white individuals, demonstrated that sST2 is an independent predictor of long-term all-cause and CVD mortality in patients with stable coronary artery disease. In the Ludwigshafen risk study (45), Dieplinger et al. noticed that sST2 adds prognostic value to the well-established cardiac biomarkers such as $\mathrm{N}$-terminal prohormone of brain natriuretic peptide (NT-proBNP) and high-sensitivity (hs) cardiac troponin $\mathrm{T}$ (cTn $\mathrm{T})$. Importantly, they were also 
able to identify subsets of patients at low, intermediate, and high risk for all-cause and CVD mortality by using a simple multi-biomarker approach which combined ST-2, NTproBNP and hs-c'TnT. Finally, patients with sST2 above the cut-off value of $24.6 \mathrm{ng} / \mathrm{mL}$ at baseline displayed a 2 -fold increased risk of $\mathrm{CV}$ death (45).

Dhillon et al. measured sST2 in 677 patients admitted to the coronary care unit, showing that increased values of ST2 were strongly associated with increased mortality at 30-day, and also revealed that most of this predictive value was retained at 1-year and beyond (46). In the same study the authors provide evidence that a multi-marker strategy combining ST2, NT-proBNP and Global Registry of Acute Coronary Event (GRACE) risk score was effective to predict mortality at 30-day beyond using either marker alone.

Sabatine et al. measured ST2 at baseline in 1,239 patients with STEMI enrolled in the CLopidogrel as Adjunctive Reperfusion Therapy-Thrombolysis in Myocardial Infarction 28 (CLARITY-TIMI 28) trial. They found that an elevated value of ST2 was a strong predictor of adverse and CVD death or HF up to 30 days. The value of sST2 provided a prognostic information that was independent from traditional laboratory biomarkers such as NT-proBNP (47).

Kohli measured sST2 in 6,560 patients from the Metabolic Efficiency with Ranolazine for Less Ischemia in the Non-ST-Elevation ACS Thrombolysis in Myocardial Infarction 36 (MERLIN-TIMI 36) study, a placebocontrolled trial. It was hence concluded that an increased value of sST2 was strongly associated with adverse outcomes at 30 days and 1 year. Furthermore, ST2 improved discrimination of risk for CVD or HF at 30 days and 1 year when added to established clinical factors and clinical biomarkers such as cardiac troponins and brain natriuretic peptide (BNP) (48).

Eggers evaluated SST2 at randomization and after 24, 48, and 72 hours in 403 non-ST-elevation ACS (NSTE-ACS) patients, demonstrating that sST2 levels were increased in these patients and also strongly predictive for mortality at 1 year after the index event. sST2 were otherwise largely independent of $\mathrm{CV}$ comorbidities or risk factors including age, diabetes or renal function (49).

\section{GDF-15}

GDF-15, a $12-\mathrm{kDa}$ secreted protein belonging to the transforming growth factor- $\beta$ (TGF- $\beta$ ) cytokine family, is a recently discovered protein that is also involved on cardiac hypertrophy and apoptosis (50). TGF- $\beta$ constitutes a superfamily of cytokines that exert prominent functions in adult tissue homeostasis and adaptation by regulating cell survival, proliferation, and differentiation. In response to pathologic or environmental stress, GDF-15 production may suddenly increase. Increases or decreases in production of TGF- $\beta s$ have been associated with a number of diseases, including neurodegenerative disorders and atherosclerosis (51). Several recent data have contributed to raise the interest around the use of GDF-15 as a biomarker for diagnosis, prognosis, and/or risk stratification of ACS. Under physiologic conditions, GDF-15 is expressed in low to moderate levels in most healthy tissues. Nevertheless, GDF-15 values may increase in response to pathological stress associated with inflammation or tissue damage, and an overexpression has been found in many malignancies including breast, melanoma, colorectal, pancreatic and prostate cancer (50-52).

It has been well established that TGF- $\beta$ inhibits the proliferation and migration of smooth-muscle and endothelial cells, and low levels of TGF- $\beta$ are commonplace in patients with atherosclerosis (53). As an emerging biomarker, GDF-15 shows promise because its values were found to be increased in early subclinical disease, retaining prognostic utility for CVD events and mortality. Previous studies showed that GDF-15 predicts adverse outcomes in patients with acute chest pain, after myocardial infarction or in patients with chronic angina. Increased circulating GDF-15 concentrations have been associated with an enhanced risk of future adverse CVD events in elderly women (54).

Hagström et al. measured GDF-15 in 16,876 patients, and showed that GDF-15 is independently associated with an increased risk of spontaneous myocardial infarction. Moreover, the GDF-15 level in patients with ACS was found to be independently associated with an enhanced risk of stroke and strongly correlated with an increased risk of CVD and total mortality in ACS patients (55).

Dominguez-Rodriguez measured GDF-15 in 502 patients, demonstrating that increased values of GDF-15 predicted subsequent CVD events after 2-year of followup. A significant and positive association between the syntax score and GDF-15 values was also noticed (56).

Wollert et al. also observed that GDF-15 was a potential promising tool for risk stratification and therapeutic decision making in patients with NSTE-ACS (57).

\section{Future perspectives}

The leading biomarkers of myocardial fibrosis, namely 
sST2 and GAL-3 are not only predictive of hospitalization and death in patients with ACS but also additive to others well-established cardiac biomarkers (i.e., natriuretic peptide levels and cardiac troponins) in prognostication of these conditions. There is consistent and striking improvement in risk stratification when $\mathrm{SST} 2$ and BNP are combined. Whether routine measurement of ST2 either alone or as part of a multimarker strategy can facilitate improved management and outcomes in acute coronary disease remains unknown and hence requires additional scrutiny. A final perspective is indeed represented by proteomics studies. A number of separate but often converging pathways are implicated in the pathogenesis of acute coronary disease, so that future prospective investigations specifically aimed at characterizing a fingerprint pattern in patients developing ACS will help to identify innovative biomarkers and disclose new data about the pathogenesis of this challenging pathology (7).

The recent development of neprilysin inhibitors for the treatment of congestive HF deserves a special mention. The assessment of different forms of BNP, which substantially include the active molecule BNP and the degradation fragment NT-proBNP, is now regarded as a mainstay for the diagnosis and for monitoring HF. However, the predictable introduction into clinical practice of neprilysin inhibitors such as LCZ696 has raised important questions regarding the reliability of measuring only one between BNP and NT-proBNP (58). Since the former biomarker is actively degraded by neprilysin but is the active part of the molecule, whereas NT-proBNP is resistant from neprilysin degradation but does not possess biological activity in vivo, emerging evidence attests that the measurement of both molecules may be more suited for optimal monitoring of $\mathrm{HF}$ in patients undergoing treatment with neprilysin inhibitors (58).

\section{Acknowledgements}

None.

\section{Footnote}

Conflicts of Interest: The authors have no conflicts of interest to declare.

\section{References}

1. Lippi G, Filippozzi L, Salvagno GL, et al. Increased mean platelet volume in patients with acute coronary syndromes. Arch Pathol Lab Med 2009;133:1441-3.

2. Goff DC Jr, Lloyd-Jones DM, Bennett G, et al. 2013 ACC/AHA guideline on the assessment of cardiovascular risk: a report of the American College of Cardiology/ American Heart Association Task Force on Practice Guidelines. Circulation 2014;129:S49-73.

3. Siemelink MA, Zeller T. Biomarkers of coronary artery disease: the promise of the transcriptome. Curr Cardiol Rep 2014;16:513.

4. Lippi G. Biomarkers: Novel troponin immunoassay for early ACS rule-out. Nat Rev Cardiol 2016;13:9-10.

5. Lippi G, Franchini M, Cervellin G. Diagnosis and management of ischemic heart disease. Semin Thromb Hemost 2013;39:202-13.

6. Cervellin G, Mattiuzzi C, Bovo C, et al. Diagnostic algorithms for acute coronary syndrome-is one better than another? Ann Transl Med 2016;4:193.

7. Lippi G, Cervellin G. Risk assessment of post-infarction heart failure. Systematic review on the role of emerging biomarkers. Crit Rev Clin Lab Sci 2014;51:13-29.

8. Casagranda I, Cavazza M, Clerico A, et al. Proposal for the use in emergency departments of cardiac troponins measured with the latest generation methods in patients with suspected acute coronary syndrome without persistent ST-segment elevation. Clin Chem Lab Med 2013;51:1727-37.

9. Lippi G. Biomarkers of myocardial ischemia in the emergency room: cardiospecific troponin and beyond. Eur J Intern Med 2013;24:97-9.

10. Lippi G, Salvagno GL, Robuschi F, et al. Influence of dipyridamole stress echocardiography on galectin-3, aminoterminal B-type natriuretic peptide (NT-proBNP) and high-sensitivity troponin T. Acta Cardiol 2014;69:377-83.

11. Krześlak A, Lipińska A. Galectin-3 as a multifunctional protein. Cell Mol Biol Lett 2004;9:305-28.

12. Houzelstein D, Gonçalves IR, Fadden AJ, et al. Phylogenetic analysis of the vertebrate galectin family. Mol Biol Evol 2004;21:1177-87.

13. de Boer RA, Yu L, van Veldhuisen DJ. Galectin-3 in cardiac remodeling and heart failure. Curr Heart Fail Rep 2010;7:1-8.

14. Chen SC, Kuo PL. The Role of Galectin-3 in the Kidneys. Int J Mol Sci 2016;17.

15. Drickamer K, Taylor ME. Biology of animal lectins. Annu Rev Cell Biol 1993;9:237-64.

16. Kasai K, Hirabayashi J. Galectins: a family of animal lectins that decipher glycocodes. J Biochem 1996;119:1-8. 
17. Cooper DN. Galectinomics: finding themes in complexity. Biochim Biophys Acta 2002;1572:209-31.

18. Barondes SH, Castronovo V, Cooper DN, et al. Galectins: a family of animal beta-galactoside-binding lectins. Cell 1994;76:597-8.

19. Ochieng J, Furtak V, Lukyanov P. Extracellular functions of galectin-3. Glycoconj J 2004;19:527-35.

20. Newlaczyl AU, Yu LG. Galectin-3--a jack-of-all-trades in cancer. Cancer Lett 2011;313:123-8.

21. Liu FT, Rabinovich GA. Galectins: regulators of acute and chronic inflammation. Ann N Y Acad Sci 2010;1183:158-82.

22. Salvagno GL, Schena F, Gelati M, et al. The concentration of high-sensitivity troponin I, galectin-3 and NT-proBNP substantially increase after a 60-km ultramarathon. Clin Chem Lab Med 2014;52:267-72.

23. Elola MT, Wolfenstein-Todel C, Troncoso MF, et al. Galectins: matricellular glycan-binding proteins linking cell adhesion, migration, and survival. Cell Mol Life Sci 2007;64:1679-700.

24. Takenaka Y, Fukumori T, Raz A. Galectin-3 and metastasis. Glycoconj J 2004;19:543-9.

25. Lippi G, Cervellin G, Sanchis-Gomar F. Galectin-3 in atrial fibrillation: Simple bystander, player or both? Clin Biochem 2015;48:818-22.

26. Ho JE, Gao W, Levy D, et al. Galectin-3 is Associated with Restrictive Lung Disease and Interstitial Lung Abnormalities. Am J Respir Crit Care Med 2016;194:77-83.

27. Sharma UC, Pokharel S, van Brakel TJ, et al. Galectin-3 marks activated macrophages in failureprone hypertrophied hearts and contributes to cardiac dysfunction. Circulation 2004;110:3121-8.

28. McCullough PA. Practical experience using galectin-3 in heart failure. Clin Chem Lab Med 2014;52:1425-31.

29. Meijers WC, van der Velde AR, Pascual-Figal DA, et al. Galectin-3 and post-myocardial infarction cardiac remodeling. Eur J Pharmacol 2015;763:115-21.

30. Suarez G, Meyerrose G. Heart failure and galectin 3. Ann Transl Med 2014;2:86.

31. Ho JE, Liu C, Lyass A, et al. Galectin-3, a marker of cardiac fibrosis, predicts incident heart failure in the community. J Am Coll Cardiol 2012;60:1249-56.

32. Winter MP, Wiesbauer F, Alimohammadi A, et al. Soluble galectin-3 is associated with premature myocardial infarction. Eur J Clin Invest 2016;46:386-91.

33. Writing Committee Members, Yancy CW, Jessup M, et al. 2013 ACCF/AHA guideline for the management of heart failure: a report of the American College of Cardiology Foundation/American Heart Association Task Force on practice guidelines. Circulation 2013;128:e240-327.

34. Tsai TH, Sung PH, Chang LT, et al. Value and level of galectin-3 in acute myocardial infarction patients undergoing primary percutaneous coronary intervention. J Atheroscler Thromb 2012;19:1073-82.

35. Maiolino G, Rossitto G, Pedon L, et al. Galectin-3 predicts long-term cardiovascular death in high-risk patients with coronary artery disease. Arterioscler Thromb Vasc Biol 2015;35:725-32.

36. Lippi G, Salvagno GL, Bonfanti L, et al. Relationship between serum galectin-3 values and demographical or biochemical variables in patients without acute coronary syndrome. Int J Cardiol 2014;171:270-1.

37. Falcone C, Lucibello S, Mazzucchelli I, et al. Galectin-3 plasma levels and coronary artery disease: a new possible biomarker of acute coronary syndrome. Int J Immunopathol Pharmacol 2011;24:905-13.

38. Gucuk Ipek E, Akin Suljevic S, Kafes H, et al. Evaluation of galectin-3 levels in acute coronary syndrome. Ann Cardiol Angeiol (Paris) 2016;65:26-30.

39. Singsaas EG, Manhenke CA, Dickstein K, et al. Circulating Galectin-3 Levels Are Increased in Patients with Ischemic Heart Disease, but Are Not Influenced by Acute Myocardial Infarction. Cardiology 2016;134:398-405.

40. Mueller T, Jaffe AS. Soluble ST2--analytical considerations. Am J Cardiol 2015;115:8B-21B.

41. Klemenz R, Hoffmann S, Werenskiold AK. Serum- and oncoprotein-mediated induction of a gene with sequence similarity to the gene encoding carcinoembryonic antigen. Proc Natl Acad Sci U S A 1989;86:5708-12.

42. Mueller T, Dieplinger B. The Presage(®) ST2 Assay: analytical considerations and clinical applications for a high-sensitivity assay for measurement of soluble ST2. Expert Rev Mol Diagn 2013;13:13-30.

43. Weinberg EO, Shimpo M, De Keulenaer GW, et al. Expression and regulation of ST2, an interleukin-1 receptor family member, in cardiomyocytes and myocardial infarction. Circulation 2002;106:2961-6.

44. Demyanets S, Speidl WS, Tentzeris I, et al. Soluble ST2 and interleukin-33 levels in coronary artery disease: relation to disease activity and adverse outcome. PLoS One 2014;9:e95055.

45. Dieplinger B, Egger M, Haltmayer M, et al. Increased soluble ST2 predicts long-term mortality in patients with stable coronary artery disease: results from the Ludwigshafen risk and cardiovascular health study. Clin Chem 2014;60:530-40.

46. Dhillon OS, Narayan HK, Khan SQ, et al. Pre-discharge 
risk stratification in unselected STEMI: is there a role for ST2 or its natural ligand IL-33 when compared with contemporary risk markers? Int J Cardiol 2013;167:2182-8.

47. Sabatine MS, Morrow DA, Higgins LJ, et al.

Complementary roles for biomarkers of biomechanical strain ST2 and N-terminal prohormone B-type natriuretic peptide in patients with ST-elevation myocardial infarction. Circulation 2008;117:1936-44.

48. Kohli P, Bonaca MP, Kakkar R, et al. Role of ST2 in nonST-elevation acute coronary syndrome in the MERLINTIMI 36 trial. Clin Chem 2012;58:257-66.

49. Eggers KM, Armstrong PW, Califf RM, et al. ST2 and mortality in non-ST-segment elevation acute coronary syndrome. Am Heart J 2010;159:788-94.

50. Ago T, Sadoshima J. GDF15, a cardioprotective TGF-beta superfamily protein. Circ Res 2006;98:294-7.

51. Kempf T, Eden M, Strelau J, et al. The transforming growth factor-beta superfamily member growthdifferentiation factor-15 protects the heart from ischemia/ reperfusion injury. Circ Res 2006;98:351-60.

52. Ek WE, Hedman ÅK, Enroth S, et al. Genome-wide DNA methylation study identifies genes associated with the cardiovascular biomarker GDF-15. Hum Mol Genet 2016;25:817-27.

Cite this article as: Salvagno GL, Pavan C. Prognostic biomarkers in acute coronary syndrome. Ann Transl Med 2016;4(13):258. doi: 10.21037/atm.2016.06.36
53. Blobe GC, Schiemann WP, Lodish HF. Role of transforming growth factor beta in human disease. $\mathrm{N}$ Engl J Med 2000;342:1350-8.

54. Brown DA, Breit SN, Buring J, et al. Concentration in plasma of macrophage inhibitory cytokine-1 and risk of cardiovascular events in women: a nested case-control study. Lancet 2002;359:2159-63.

55. Hagström E, James SK, Bertilsson M, et al. Growth differentiation factor-15 level predicts major bleeding and cardiovascular events in patients with acute coronary syndromes: results from the PLATO study. Eur Heart J 2016;37:1325-33.

56. Dominguez-Rodriguez A, Abreu-Gonzalez P, Avanzas P, et al. Effect of Serum Growth Differentiation Factor-15 and the Syntax Score on 2-Year Outcomes in Patients With Acute Coronary Syndrome. Am J Cardiol 2016;117:1569-74.

57. Wollert KC, Kempf T, Lagerqvist B, et al. Growth differentiation factor 15 for risk stratification and selection of an invasive treatment strategy in non ST-elevation acute coronary syndrome. Circulation 2007;116:1540-8.

58. Lippi G, Sanchis-Gomar F. Monitoring B-type natriuretic peptide in patients undergoing therapy with neprilysin inhibitors. An emerging challenge? Int J Cardiol 2016;219:111-4. 